\title{
Before and after Analysis of Enhanced Recovery Studies
}

\author{
Christopher Malgieri \\ Department of Anesthesiology, Rhode Island Hospital, The Warren Alpert Medical School of Brown University, \\ Providence, RI, USA
}

\section{Dear Editor,}

I read with great interest the article by Perinel and colleagues in a recent issue of the journal [1]. The authors performed a before/after study with a contemporary control group of 172 patients undergoing pancreatectomy and concluded that the implementation of an enhanced recovery after surgery (ERAS) program was safe and effective after pancreatectomy, with a high compliance rate. In addition, the hospital length of stay was significantly reduced without compromising morbidity. The authors should be applauded for performing a well-designed study on an important topic (e.g., ERAS) in patients undergoing digestive surgery $[2,3]$. Moreover, the need to improve quality of care while reducing costs is the holy grail of modern medicine [4].

Although the study by Perinel and colleagues was performed well, there are several critical points that need to be clarified to determine the validity of the results. First, it is important to know if all patients received comparable postoperative analgesic regimen as this can alter the study results. Appropriate analgesic management is one of the most important components of ERAS, but the authors do not describe in detail the differences in intraoperative and postoperative analgesic regimens between the hospitals

karger@karger.com

(C) 2020 S. Karger AG, Basel

www.karger.com/dsu

Karger"
[5]. It is possible that the use of regional anaesthetic techniques alone has resulted in greater postoperative pain control, reduction of opioid consumption, and faster return of bowel functions. Last, the authors utilized a Cox regression analysis but did not provide evidence that the time-to-event data are proportional, and this may alter the results and interpretation of the study [6]. I would welcome comments by the authors as this would help further support the findings of this important clinical study.

\section{Disclosure Statement}

The author has no conflicts of interest to declare.

\section{Funding Sources}

The author did not receive any funding.

\section{Author Contribution}

C.M. contributed to the study's concept, writing the manuscript, and critical review of the manuscript. 


\section{References}

1 Perinel J, Duclos A, Payet C, Bouffard Y, Lifante JC, Adham M. Impact of enhanced recovery program after surgery in patients undergoing pancreatectomy on postoperative outcomes: a controlled before and after study. Dig Surg. 2019 Feb 22:37(1):47-55.

2 Arrick L, Mayson K, Hong T, Warnock G. Enhanced recovery after surgery in colorectal surgery: impact of protocol adherence on patient outcomes. J Clin Anesth. 2019;55:712.
3 Slieker JC, Clerc D, Hahnloser D, Demartines N, Hübner M. Prospective evaluation of discharge trends after colorectal surgery within an enhanced recovery after surgery pathway. Dig Surg. 2017;34(4):298-304.

4 Serdiuk AA, Urman RD. Quality, cost and teamwork: adding the human element to perioperative services. Proceedings of the association of anesthesia clinical directors (AACD) annual perioperative summit, 2019. J Clin Anesth. 2019;58:55-6.
5 Markham T, Wegner R, Hernandez N, Lee JW, Choi W, Eltzschig HK, et al. Assessment of a multimodal analgesia protocol to allow the implementation of enhanced recovery after cardiac surgery: retrospective analysis of patient outcomes. J Clin Anesth. 2019;54:7680.

6 McGregor DE, Palarea-Albaladejo J, Dall PM, Hron K, Chastin S. Cox regression survival analysis with compositional covariates: application to modelling mortality risk from 24-h physical activity patterns. Stat Methods Med Res. 2019 Jul 25:29(5):1447-65. 